\title{
Subspace Method-based Blind SNR Estimation for Communication between Orbiters in Mars Exploration
}

\author{
Ze-Zhou SUN ${ }^{1,2}$, Cheng-Hua WANG ${ }^{1}$ and Xiao-Fei ZHANG ${ }^{1, a}$ \\ ${ }^{1}$ College of Electronic and Information Engineering, Nanjing University of Aeronautics and Astronautics, Nanjing, \\ 210016, China \\ ${ }^{2}$ Department of overall design, the China Academy of space technology, Beijing, China
}

\begin{abstract}
In Mars exploration the effective time of communication between orbiters is short, and the relative distance and gesture between them change fast. The signal to noise ratio (SNR) estimation is required in receiver to change adaptively the data rate in the communication system. Therefore, SNR estimation is a key technique in adaptive data transmission. We propose a blind SNR estimation for communication between orbiters in Mars exploration via subspace method. The subspace method has better SNR estimation than some conventional SNR estimation algorithms. Numerical simulations demonstrate the effectiveness and improvement of the proposed algorithm.
\end{abstract}

\section{Introduction}

The long distance between Mars and Earth causes long transmission time delay and signal attenuation. In Mars exploration the effective time of communication between orbiters is short, and the relative distance and gesture between them change fast $[1,2,3]$. The signal to noise ratio (SNR) estimation is required in receiver to change adaptively the data rate of the communication system. SNR estimation approach contains frequency domain method and time domain method. Time domain method contains data aided (DA) method and non-data aided (NDA) method [4]. DA method has higher estimation accuracy than NDA method, but it needs the insertion of the training sequence, which lowers the transmission efficiency. In time domain method, SNR estimation algorithms based on DA contain maximum likelihood (ML), minimum mean square error (MMSE) [5, 6], split symbol moments estimator (SSME) [7, 8], and the separation of signal and noise using higher-order cumulants [9]. SNR estimation algorithms based on NDA are second- and fourth-order moments (M2M4) [10, 11], signal-to-variation ratio (SVR) [12], squared signal-to-noise variance (SNV) [13], and data fitting (DF) [14]. Classic frequency domain method is based on the flat character of power spectrum of white noise, which is suitable for SNR estimation of AWGN channel, but not for colored-noise environment.

This paper intends to develop a blind SNR estimation for communication between orbiters in Mars exploration via subspace method, which not only achieves accurate estimate result. The subspace method has better SNR estimation than some conventional SNR estimation algorithms including DF, SVR, SNV and M2M4.

\footnotetext{
a Corresponding author: fei_zxf@163.com
} 
The reminder of this paper is structured as follows. The data model is given in Section 2. Section 3 introduces the blind SNR estimation algorithm via subspace method. Section 4 presents the simulation results, and the conclusions are drawn in Section 5.

\section{Data model}

In complex additive white Gaussian noise (AWGN) channel, the received signals can be represented as follows:

$$
r_{k}=x_{k}+n_{k}
$$

where $n_{k}$ is a zero-mean complex AWGN sample with a variance of $\sigma_{n}^{2}$. When the sampling time is $t$, the noise column vector consisting of $L$ samples is $\mathbf{n}(t)=\left[n_{t}, n_{t-1}, \cdots, n_{t-L+1}\right]^{T}$, where $(\bullet)^{T}$ denotes the transpose. The second-order moment of the noise vector can be written as

$$
E\left[\mathbf{n}(t) \mathbf{n}^{\mathrm{H}}(I)\right]=\sigma_{n}^{2} I \delta_{t l}
$$

where $E[\bullet]$ denotes the mathematical expectation, the upper-case symbol $\mathrm{H}$ is the Hermitian transpose, and $I$ is the $L \times L$ identity matrix. Function $\delta_{t l}$ has the value of 1 only when $t=l$, while under other circumstances it has the value of $0 . X_{k}$ is the bandpass signal at the carrier frequency $f_{c}$, i.e.,

$$
x_{k}=\tilde{x} e^{-j\left(2 \pi f_{c} k / f_{s}+\theta\right)}
$$

$\tilde{x}_{k}$ is the complex equivalent baseband signal. In terms of MPSK signal. In terms of MQAM signal, $\tilde{x}_{k}=A_{l} e^{j 2 \pi l / M}(l=0, \ldots, M-1), A_{l} \in C$ represents the corresponding amplitude. $\theta$ is the initial phase of the carrier, and $f_{s}$ is the sampling rate. Let the signal power and the noise power be $P_{x}$ and $P_{n}$, respectively, so the SNR can be written as [14]

$$
\rho=10 \lg \left(P_{x} / P_{n}\right)
$$

\section{Blind SNR estimation algorithm via subspace method}

Assume that the receiving signal has $N$ numbers of symbols. We sample $L$ times for each symbol, then the $n$th symbol after sampling can be expressed as $\mathbf{r}_{n}(t)=\left[r_{n}(t), r_{n}(t-1), \cdots, r_{n}(t-L+1)\right]^{T}$. The receiving signal is a $L \times N$ matrix 


$$
\mathbf{R}=\left[\begin{array}{cccc}
\mathbf{r}_{1}(t) & \mathbf{r}_{2}(t) & \mathrm{L} & \mathbf{r}_{N}(t) \\
\mathbf{r}_{1}(t-1) & \mathbf{r}_{2}(t-1) & \mathrm{L} & \mathbf{r}_{N}(t-1) \\
\mathrm{L} & \mathrm{L} & \mathrm{L} & \mathrm{L} \\
\mathbf{r}_{1}(t-L+1) & \mathbf{r}_{2}(t-L+1) & \mathrm{L} & \mathbf{r}_{N}(t-L+1)
\end{array}\right]
$$

The covariance matrix of it is $\mathbf{R}_{r r}=E\left[\mathbf{r}_{n}(t) \mathbf{r}_{n}^{H}(t)\right]$. Assume the signal and the noise are uncorrelated. $\mathbf{R}_{r r}$ can be expressed as

$$
\mathbf{R}_{r r}=\mathbf{R}_{x x}+\mathbf{R}_{n n}
$$

According to the eigenvalue decomposition theorem, $R_{r r}$ can be denoted by[15]

$$
\mathbf{R}_{r r}=\mathbf{A} \Sigma \mathbf{A}^{H}
$$

where $\mathbf{A}$ consists of the eigenvectors. The diagonal matrix $\Sigma=\operatorname{diag}\left(b_{1}, b_{2}, \cdots, b_{L}\right)$ represents the eigenvalues of $\mathbf{R}_{r r}$, where $b_{1} \geq b_{2} \geq \cdots b_{L}$. From Eq. 2 we know that the autocorrelation matrix of white noise $\mathbf{R}_{n n}$ satisfies

$$
\mathbf{R}_{n n}=\sigma_{n}^{2} \mathbf{I}
$$

Then the eigenvalues of $\mathbf{R}_{r r}$ is in the form of

$$
b_{i}=\left\{\begin{array}{l}
\sigma_{x_{i}}^{2}+\sigma_{n}^{2}, \quad 1 \leq i \leq p \\
\sigma_{n}^{2}, \quad p+1 \leq i \leq L
\end{array}\right.
$$

where $\sigma_{x_{i}}^{2}$ denotes the signal power of the $i$ th eigenvector. The signal subspace is the subspace spanned by the first $p$ eigenvectors, while the noise subspace is the subspace spanned by the last $L-p$ eigenvectors. And the noise power $P_{n}$ is $L$ times of $\sigma_{n}^{2}$. When the signal subspace dimension $p$ is fixed, the SNR estimates could be obtained via calculation of $\sigma_{n}^{2}$ and $P_{n}$.

The noise power can be obtained by

$$
\hat{P}_{n}=\sum_{i=p+1}^{L} b_{i} /(L-p)
$$

Then, the source signal power is

$$
\hat{P}_{s}=\sum_{i=1}^{p}\left(b_{i}-P_{n}\right)
$$

We can adopt the Minimum Description Length (MDL) criterion [16] to estimate the signal 
subspace dimension $p$, while $p$ is known as the number of source signal in this paper. Actually it is hard to get the perfect value of correlated matrix $\mathbf{R}_{r r}$, since we can only estimate it via the limited-length received signals. Assume there are $N$ observation vectors, and the sampled covariance matrix is

$$
\hat{\mathbf{R}}_{r r}=\frac{1}{N} \sum_{n=1}^{N} \mathbf{r}_{n}(t) \mathbf{r}_{n}^{H}(t)
$$

It is proven that the eigenvalues of $\hat{\mathbf{R}}_{r r}$ defined by Eq. 11 are the maximum likelihood of eigenvalues of $\mathbf{R}_{r r}$.

The steps of blind SNR estimation algorithm via subspace method are as follows:

Step1: Obtain the covariance matrix estimate $\hat{\mathbf{R}}_{r r}$ from the received signal.

Step2: Compute the ED of $\hat{\mathbf{R}}_{r r}$ according to Eq. 6 .

Step3: Sort the eigenvalues $b_{i}$ in descending orders.

Step4: Estimate the noise power and the signal power according to Eq. 9 and Eq. 10, respectively; Step5: Obtain the SNR estimate according to Eq. 4.

\section{Simulation results}

We use Monte Carlo simulations to assess the performance of the proposed algorithms. Use $\rho$ to represent the actual value of SNR, and $\hat{\rho}_{k}$ is the estimate of $\rho$ in the $l$ th Monte Carlo trial, so the RMSE can be written as

$$
R M S E\{\hat{\rho}\}=\sqrt{\frac{1}{M} \sum_{k=1}^{M}\left(\hat{\rho}_{k}-\rho\right)^{2}}
$$

where $M$ is the number of Monte Carlo simulations.

In simulations, we choose QPSK signal over AWGN channel. The roll-off root of the raised cosine filter factor is 0.5 and tap number is 65 . The number of samples $L$ is 16 , the number of symbols $N$ is 300 , and the number of Monte Carlo simulations $M$ is 500 .

Figure.1 demonstrates the SNR estimate of subspace method. SNR estimate gradually approaches to real SNR as the real SNR increasing.

The RMSE of SNR estimate via subspace method under different numbers of symbols is shown in Figure. 2. Under the same SNR, the greater the number of the symbols is, the smaller the RMSE of SNR estimate is.

We compare our proposed algorithm against the DF, M2M4, SVR and SNV, which is shown in Figure.3. It is shown in Figure.3 that the SNR estimation performance of subspace tracking method is the best among the algorithms. 


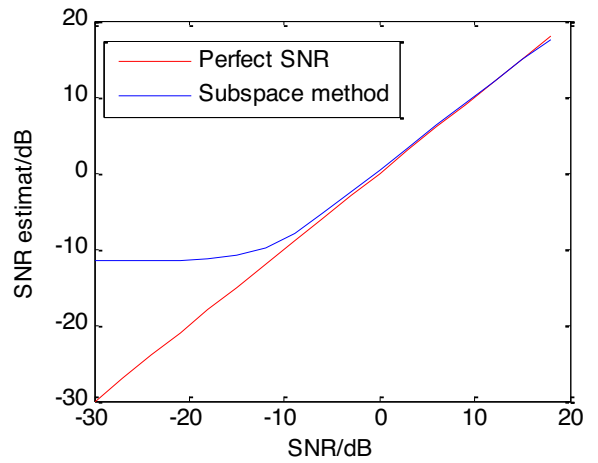

Figure. 1 SNR estimate of subspace method

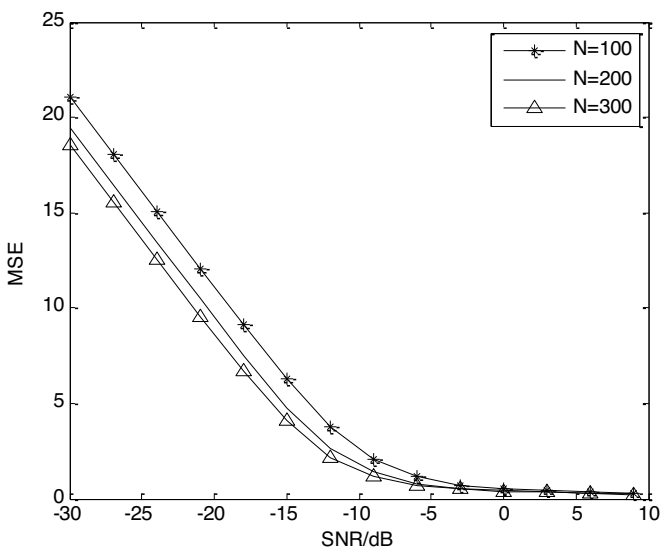

Figure. 2 RMSE of SNR estimate under different numbers of symbols

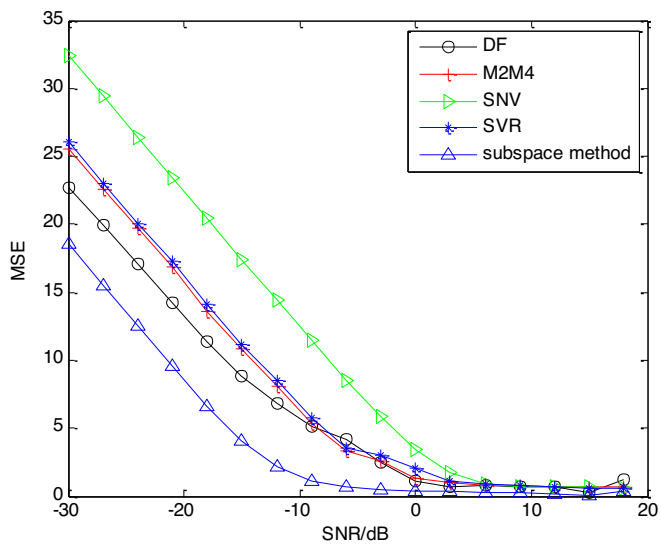

Figure. 3 Estimation performance comparison of different algorithms $(\mathrm{N}=300)$ 


\section{Conclusions}

A blind SNR estimation for communication between orbiters in Mars exploration via subspace method is proposed in this paper. The subspace method has better SNR estimation than some conventional SNR estimation algorithms.

\section{Acknowledgement}

This work is supported by China NSF Grants (61371169), Qing Lan Project, and priority academic program development of Jiangsu high education institutions.

\section{References}

1. $\mathrm{Ru} \mathrm{J}$ X. Numerical analysis of Mars exploration orbit[J]. Science in China (Series E: Technological Sciences), 39(03): 528-534. (2009)

2. Guo H L, Wang Z S, Qu G J. Flight principle of Mars exploration and launch timing analysis [J]. Science in China (Series E: Technological Sciences), 39(03): 535-543. (2009)

3. Zhang X H, Liu Z S. Trajectory Design and Optimization of Mars Satellite. MISSILEAND SPACE VEHCILE. NO.2. 15-23. (2008)

4. Fu W J, Jiang J S, Wang X R, Liu S Y. New SNR Estimation Methods in Digital QPSK Receiver [J]. Journal of Electronics \& Information Technology, 29, No.2, 255-258. (2007)

5. Wiesel A, Goldberg J, Messer H. Non-Data-Aided Signal-to-Noise-Ratio Estimation[C]// 2002 IEEE International Conference on Communications, 197-201. (2002)

6. Li B, Difazio R, Zeira A. A low bias algorithm to estimate negative SNRs in an AWGN channel [J]. IEEE Communications Letters, 6(11):469-471. (2002)

7. Shah B, Hinedi S. The split symbol moments SNR estimator in narrow-band channels [J]. IEEE Transactions on Aerospace \& Electronic Systems, 26(5):737-747. (1990)

8. M. K. Simon and S. Dolinar. Signal-to-noise ratio estimation for autonomous receiver operation [J]. IEEE GLOBECOM 04:pp: 282-287 Dallas, TX. (2004)

9. Tugnait J K, Gummadavelli U. Blind channel estimation and deconvolution in colored noise using higher-order cumulants [C]// Advanced Signal Processing: Algorithms, Architectures, and Implementations, 106-125. (1994)

10. Ren G L, Chang Y L, Zhang H. A new SNR estimator for QPSK Modulation in an AWGN channel [J]. IEEE Transactions Circuits and Systems II: Express Briefs, 52(6): 336-338. (2005)

11. Matzner R, Englberger F. An SNR estimation algorithm using fourth-order moments [C]// 1994 IEEE International Symposium on Information Theory, IEEE. (1994)

12. Salman T, Badawy A, Elfouly T.M, Khattab T. Non-data-aided SNR Estimation for QPSK Modulation in AWGN Channel [C]. IEEE Wireless and Mobile Computing, Networking and Communications Conference, Larnaca, 611-616. (2014)

13. Gilchriest C E. Signal-to-noise monitoring [J]. JPL Space Programs Summary, 4: 169-184. (1966)

14. Hua Xu, Zupeng Li, Hui Zheng. A non-data-aided SNR estimation algorithm for QAM signals [C]. IEEE Circuits and Systems, International Conference on Communications, Chengdu,999-1003. (2004)

15. Ramakrishna D, Mandayam N B, Yates R D. Subspace-based SIR estimation for CDMA cellular systems [J]. IEEE Transactions on Vehicular Technology, 49(5):1732-1742. (2000)

16. Wax M. and Kailath T. Detection of signals by information theoretical criteria. IEEE Trans. on ASSP, 33(2): 387-392. (1985) 